\title{
Hysteroscopy and endometrial cancer. Diagnosis and influence on prognosis
}

\author{
N. Koutlaki • M. Dimitraki • S. Zervoudis • P. Skafida • \\ I. Nikas • J. Mandratzi • A. Liberis • V. Liberis
}

Received: 6 June 2010 / Accepted: 6 July 2010 / Published online: 28 July 2010

(C) Springer-Verlag 2010

\begin{abstract}
Diagnostic hysteroscopy is a simple procedure that can provide a good visualization of the whole uterine cavity without cervical dilation and usually without anesthesia. It has been proposed as an office method for diagnosis of endometrial disorders, including cancer. Hysteroscopy, especially combined with endometrial biopsy, has high diagnostic accuracy. However, concerns have been raised about the potential for hysteroscopy to flush malignant endometrial cells into the peritoneal cavity through the fallopian tubes and to cause dissemination of malignant cells into the abdominal cavity through the fallopian tubes from uteri containing endometrial carcinoma. Moreover, some controversy also remains about the functional viability of these cells and their potential to cause metastases.
\end{abstract}

Keywords Hysteroscopy · Endometrial cancer .

Peritoneal cytology $\cdot$ Dissemination $\cdot$ Cell viability

\section{Background}

The development of new methods for the endometrial investigation has allowed discovering the endometrial cancer in early stages, even in asymptomatic women.

N. Koutlaki $(\square) \cdot$ M. Dimitraki $\cdot$ P. Skafida $\cdot$ I. Nikas

J. Mandratzi $\cdot$ A. Liberis $\cdot$ V. Liberis

Department of Obstetrics and Gynecology, University Hospital

of Alexandroupolis, Democritus University of Thrace,

Alexandroupolis 68100, Greece

e-mail: nikoleta_koutlaki@yahoo.gr

S. Zervoudis

Lito Maternity Hospital,

Athens, Greece
Among the methods used in endometrial disorder screening, diagnostic hysteroscopy is a simple procedure that can provide a good visualization of the whole uterine cavity without cervical dilation and usually without anesthesia. However, one important limitation about the office hysteroscopy usage is the lack of studies correlating the visual aspects of the endometrial lesion and the histology, as the subjective hysteroscopic appearance, based on surgeon experience, does not allow distinguishing all cases of endometrial carcinoma from benign endometrial disease $[1,2]$. Several investigators, who suggest the use of hysteroscopy for diagnosis of endometrial cancer, admit that a carcinoma with polypoid appearance can be confused with a benign endometrial polyp [3,4]. According to other authors [5] differential diagnosis among hyperplasia or endometrial carcinoma using the subjective hysteroscopic view could be made in only $60.9 \%$ of the cases.

Sugimoto [3] first proposed hysteroscopic classification of endometrial carcinoma, specifying the four types of tumor growth: (a) polypoid, (b) nodular, (c) papillary, and (d) ulcerated type.

Also, Sugimoto in his study [3], which included 53 patients, described the polypoid, nodular, and papillary types as exophytic and circumscribed disease, but the ulcerated type as endophytic and diffuse [6].

It should be mentioned that hysteroscopic appearance of endometrial carcinoma is quite typical. The visual diagnosis is generally based on the presence of a gross distortion of endometrial cavity, because of focal or extensive nodular, polypoid, papillary, or mixed patterns of neoplastic growth. Focal necrosis, friable consistency, and atypical vessels are other features almost invariably associated with endometrial cancer and easily detected by hysteroscopic inspection [7]

In the study of Zola et al. [8], 21 consecutive patients with a histologically confirmed endometrial malignancy 
(cases) and 42 patients with benign endometrial disease (controls) were included. Most of the endometrial malignancies were endometrioid carcinoma (17), followed by papillary serous carcinoma (two), clear cell carcinoma (one), and mixed mullerian tumor (one). The controls were endometrial polyp (33), simple endometrial hyperplasia (four), sub-mucous leiomyoma (three), and cystic endometrial atrophy (two). Hysteroscopic findings associated with malignancy were papillary aspect, size $>1 / 2$ uterine cavity, irregular surface, mixed color, diffuse vascular arrangement, loss of branched vascularization, and discordance between the main vascular axe and the direction of the lesion growth. Ulcerated surface and anarchic vascular aspect were present only in malignant cases.

Regarding the morphologic appearance of endometrial disease, the papillary type is significantly more frequent in malignant tumors. The presence of a warty aspect, with many tentacle-like projections, suggests a pattern of fast, aggressive, and disorganized growing. The polypoid-type lesions are mostly benign, but eventually, lesions with malignant behavior grow so fast into the uterine cavity that mimic benign polypoid lesions. So, the presence of a polypoid aspect is not enough to prove benignity. The nodular lesions with smooth surface, presented as large protrusions on the endometrial wall, are associated with benign histology, in agreement with the literature [3].

Lesions bigger than a half of the uterine cavity are suggestive of malignancy. However, analysis of the lesion size as a criterion for differential diagnosis was not found in the literature, but the tumoral size may be associated with invasion and dissemination in endometrial cancer [9]. Size evaluation might be limited since hysteroscopy is subjective and it is difficult to measure structures because of distortion and magnification [8].

Irregular and ulcerated surfaces, with areas of necrosis due to the insufficient blood supply, are associated with malignant lesions. Ulcerations found only in malignant lesions probably are the endpoint of irregular surface. The lesions with velvety surface are associated with thick normal endometrium, but might be present in endometrial hyperplasia or well-differentiated malignant tumors [8].

The endometrial color is related with the vascular pattern. Pale colors suggest insufficient blood supply, like in atrophic endometrium, and are associated with benign lesions. The mixing of colors represent areas with increased vascularization and necrosis, and are associated with malignancy. The reddish color is associated with increased vascularization and could be related to normal, inflammatory, or malignant endometrial tumors. Lesions with increased vascularization on the entire surface are likely malignant, and when the increase of the vessels occurs only in some areas the lesions are usually benign. The pattern of vascularization reflects the formation of new vessels [10].
Reticular aspect is typical of capillary nets between arteries and veins and occurs in benign lesions. On the other hand, the loss of this reticular distribution, the presence of vessels without branches suggests fast angiogenesis and occurs in malignant tumors.

An interesting observation is also the relationship between the main vascular axe and the direction of the lesion growth. Benign lesions progress slowly together with the progress of their vascularization and in these cases we can see a perfect agreement between the main vascular axe and the direction of the lesion growth. Malignant tumors, probably because of their irregular growth and disorganized angiogenesis, lose that visual characteristic [8].

Generally, the hysteroscopic features associated with malignancy are: papillary aspect, size larger than a half of uterine cavity, irregular, and ulcerated surface, mixed color, diffuse vascularization with anarchical or slightly branched aspect, discordance between the main vascular axe and the direction of the lesion growth [8].These findings can be used as guide for visual diagnose during panoramic hysteroscopy.

It is clinically important to differentiate preoperatively stage IA disease from invasive, especially superficial microinvasive disease in well-differentiated endometrioid adenocarcinoma, as nonsurgical conservative management may be used in stage IA disease in premenopausal patients, including those who desire fertility or possible less extensive surgery [6].

There is little information on a possible relationship between the endoscopic appearance and the myometrial invasion of endometrial carcinoma. Taddei et al. [11] reported that endometrial extension of focal, partial, or diffuse disease estimated by hysteroscopy is poorly related to the depth of myometrial invasion. According to the study of Garuti et al. [9] on 60 patients with endometrial carcinoma, the endoscopic morphology was unrelated to the histological and surgical grades. No relationship to patient survival was found, although the extent of carcinomatous spread within the endometrial cavity was significantly related to the stage, grade, and survival. In a more recent study of Kanazawa et al. [6], the preoperative hysteroscopic appearance of 97 patients with welldifferentiated endometrioid adenocarcinoma was correlated with the histological findings of the hysterectomy specimen. According to the study, the hysteroscopic findings help in the correct selection of stage IA disease confined to the endometrium, with sensitivity and negative predictive value (NPV) more than 90\%, while the specificity and positive predictive value (PPV) were less than 65\% when calculated independently according to the growth pattern of the tumor with or without ulceration. However, when these parameters were calculated combining the tumor growth pattern and the presence of ulceration, the specificity rose to 
$75 \%$ and the PPV to $72 \%$, with no significant change in sensitivity and NPV.

Today, magnetic resonance imaging (MRI) or contrastenhanced MRI is used as a high accuracy technique for determining the depth of myometrial tumor invasion preoperatively, but not for determining the absence of myometrial invasion. It has been pointed out that there are limitations on preoperative diagnosis of early microscopic or superficial myometrial invasion even by recent advances in MRI method [12-15] (sensitivity $57-100 \%$ and PPV $75-100 \%$, in different MRI techniques $[14,15])$. Thus, hysteroscopy is valuable as an adjunct to a more complete assessment of the lack of myometrial invasion in well-differentiated endometrioid adenocarcinoma.

\section{Diagnostic accuracy of hysteroscopy}

Since the time that Gimpelson and Rappold [16] detected that hysteroscopy combined with endometrial biopsy offers higher diagnostic accuracy than dilatation and curettage $(\mathrm{D} \& \mathrm{C})$ alone, it has been widely accepted as the gold standard for the investigation of endometrial pathology. Despite the limited data on the diagnostic accuracy of hysteroscopy, it is used, with or without endometrial biopsy, as a reference method in the majority of studies.

A study with a large patient sample (1,500 women-806 promenopausal, 694 menopausal undergoing diagnostic hysteroscopy because of abnormal uterine bleeding) was the one of Garuti et al., published in 2001 [17], aiming to estimate the accuracy of hysteroscopy in predicting endometrial histopathology. Hysteroscopy showed sensitivity, specificity, NPV, and PPV of $94.2 \%, 88.8 \%, 96.3 \%$, and $83.1 \%$, respectively, in predicting normal or abnormal histopathology of endometrium. Highest accuracy was in diagnosing endometrial polyps, with sensitivity, specificity, NPV, and PPV of $95.3 \%, 95.4 \%, 98.9 \%$, and $81.7 \%$, respectively; the worst result was in estimating hyperplasia, with respective figures of $70 \%, 91.6 \%, 94.3 \%$, and $60.6 \%$. Authors reported that all failures of hysteroscopic assessment resulted from poor visualization of the uterine cavity or from underestimation or overestimation of irregularly shaped endometrium.

One of initial and more systemic assessments of the diagnostic accuracy of hysteroscopy in the diagnosis of endometrial cancer is the study of Clark et al. [18]. It is a metaanalysis of 65 primary studies, including 26,346 women (29\% were postmenopausal), assessed the diagnostic accuracy of hysteroscopy in detecting serious endometrial disease. The diagnostic reference standard was endometrial histological findings. The overall sensitivity of hysteroscopy (successful procedure) was $86.4 \%$ and specificity was $99.2 \%$ for endometrial cancer. However, as sensitivity and specificity have limited value in clinical interpretation, likelihood ratios (LRs) were used as the principal measures of diagnostic accuracy (the LRs indicate how much a given hysteroscopic finding increases or decreases the probability of having endometrial cancer or disease). The (+) LR was 60.9, and the (-) LR 0.15. The pretest probability (prevalence) increased from $3.9 \%$ to $71.8 \%$ for a positive result and decreased to $0.6 \%$ for a negative result. The high LR for a positive test result on hysteroscopy should increase most pretest probabilities over any threshold for advanced management. In contrast, the LR of 0.15 for a negative test result is not low enough to negate the need for further diagnostic testing. Hysteroscopy is thus highly accurate and thereby clinically useful in diagnosing endometrial cancer in women with abnormal uterine bleeding. However, its high accuracy relates to diagnosing cancer rather than excluding it.

A more recent study [9] evaluated the contribution of hysteroscopic imaging to the decrease of the rate of undetected endometrial carcinomas concurrent with atypical hyperplasia diagnosed by endometrial biopsy. Hysteroscopic reports of 25 menopausal patients undergoing endometrial biopsy yielding a diagnosis of atypical hyperplasia were reviewed. On the basis of this diagnosis, all patients were treated by hysterectomy, and the pathologic findings on the uterine specimen were correlated with the diagnoses obtained by hysteroscopic view. By hysteroscopic inspection, two intramucous carcinomas were underestimated as endometrial hyperplasia. This finding might be related to the early stage of neoplasias, influencing the lack of gross endometrial distortion associated with more advanced carcinomas and showing hysteroscopic features possibly overlapping some of the ones considered as specific of hyperplasia. These underestimated diagnoses led NPV (87.5\%) and sensitivity (84.6\%) of hysteroscopy to be less than optimal in excluding an endometrial carcinoma when the visual findings are consistent with endometrial hyperplasia.

In another recent study [19], hysteroscopic view was validated with histology in cases of endometrial hyperplasia and cancer in patients with abnormal uterine bleeding. Among 4,054 examinations, hysteroscopic views were compatible with cancer or hyperplasia ( $80.0 \%$ and $16.2 \%$, respectively) in the histologic cases of endometrial cancer (101 cases, 96.2\%). In endometrial hyperplasia, sensitivity of the hysteroscopic view was $56.3 \%$ (95\% CI 52.2-60.2\%), specificity was $89.1 \%$ (95\% CI 88.0-90.1\%), PPV was $48.0 \%$ (95\% CI 44.3-51.7\%), NPV was $92.0 \%$ (95\% CI 90.1-92.9\%), and accuracy was $72.7 \%$ (95\% CI 70.7-74.7\%); and in endometrial cancer, the sensitivity of the hysteroscopic view was $80.0 \%$ (95\% CI 71.1-87.2\%), specificity was $99.5 \%$ (95\% CI 99.2-99.7\%), PPV was $81.5 \%$ (95\% CI 72.7-88.5\%), NPV was $99.5 \%$ (95\% CI 99.2-99.7\%), and accuracy was $89.8 \%$ (95\% CI, 85.9-93.6\%). In the histologic cases of endometrial 
cancer, 101 (96.2\%) hysteroscopic views were compatible with cancer or hyperplasia $(80.0 \%$ and $16.2 \%$, respectively) and 97 out of $103(94.2 \%)$ hysteroscopic views with cancer findings had histologic diagnosis of cancer or hyperplasia $(81.5 \%$ and $12.6 \%$, respectively).These data declare that hysteroscopy is an accurate method for endometrial pathology investigation with good sensitivity.

Gabrielli et al. [20] in their study tried to evaluate the diagnostic accuracy of transvaginal sonography (TVS) versus hysteroscopy in detecting cervical involvement. The accuracy of hysteroscopy in detecting cervical involvement was $72 \%$, sensitivity $64 \%$, specificity $73 \%$, PPV $32 \%$, and NPV $91 \%$.

Kietlińska et al. [21] compared D\&C, hysteroscopy, TVS, MRI, and CT in cervical canal involvement evaluation. $33.3 \%$ sensitivity and $68.6 \%$ specificity is reported for hysteroscopy.

\section{Intraperitoneal spread of endometrial cancer cells with hysteroscopy. True or a myth?}

There is evidence from observational studies that liquid distension of endometrial cavity used to allow complete visualization of the fundus and ostial areas during hysteroscopy, can be associated with transtubal reflux of endometrial cells and tissue into the peritoneal cavity, particularly by use of $32 \%$ dextran as a distension medium $(42-100 \%$ detection rate of endometrial tissue in the pelvis) $[22,23]$.

There are contradictory results about the use of $\mathrm{CO}_{2}$ distension. Lo et al. [24] suggested that endometrial malignant cells were introduced into the peritoneal cavity during hysteroscopy and that positive peritoneal cytology was significantly more common among patients having hysteroscopy using normal saline (NS) rather than $\mathrm{CO}_{2}$ (14.0\% versus $1.4 \%$, OR $11.2,95 \%$ CI, $P$ 0.009). There was no statistically significant difference between the two groups of patients undergoing hysteroscopy using either $\mathrm{CO}_{2}$ or NS with regard to other prognostic factors (age, pathologic stage, International Federation of Gynecology and Obstetrics (FIGO) grading, myometrial invasion, tumor size, c ervical involvement, nodal involvement, and 2-year disease-free survival).

A prospective randomized self-controlled study by Nagele et al. [25] showed no significant difference in the spreading of endometrial cells after hysteroscopy using $\mathrm{CO}_{2}$ or NS hysteroscopy. Transtubal dissemination occured in about one quarter of patients, irrespective $\mathrm{CO}_{2}$ or NS was used for uterine distension.

There are conflicting reports about intrauterine pressure levels needed for transtubal dissemination. It has been determined that $100 \mathrm{mmHg}$ is the median intrauterine perfusion pressure for spill of dye from both fallopian tubes and that no spill occurs at pressure less than $70 \mathrm{mmHg}$ [26]. Sagawa et al. [27] found a slightly higher percentage of positive cytology in patients diagnosed with high-pressure hysteroscopy compared to patients diagnosed with low pressure hysteroscopy.

In the study of Solima et al. [28] three different methods were used to evaluate transtubal leakage during hysteroscopy: blue dye, technetium Tc $99 \mathrm{~m}$ and cytologic analysis. The solution bag of the distension medium was maintained $50 \mathrm{~cm}$ above the patient plane, so that the infusion pressure could not exceed $40 \mathrm{mmHg}(50 \mathrm{cmH} 2 \mathrm{O})$. When this uterine pressure was used, in no case did transtubal spillage occur. Transtubal spillage was observed only in two patients, in whom infusion pressure was increased by elevating the plastic bag up to $70 \mathrm{~cm}$ above the patient's plane because intrauterine bleeding was impairing visibility. The detection of the tracers in the peritoneal cavity could give more accurate information about the iatrogenic spillage of endometrial cells during hysteroscopy. According to this study, no spill of neoplastic cells occurs at pressure less than $40 \mathrm{mmHg}$. The detection of positive cytology after hysteroscopy is not a guarantee that the dissemination of transtubal neoplastic cells occurred because of the procedure [28].

Finally, according to the data of a recent study [29], diagnostic hysteroscopy significantly increases the risk of positive peritoneal cytology, but not the risk of adnexal, abdominal, or retroperitoneal lymph node metastases in patients with endometrial cancer.

\section{Positive peritoneal cytology and outcome of endometrial cancer}

Peritoneal cytology became an important determinant in the new classification system for staging of endometrial cancer (1988, FIGO). In particular, stage I patients (i.e., endometrial cancer confined to the uterus) and stage II patients (i.e., endometrial cancer invading the cervix) who demonstrated positive peritoneal cytology were upstaged to stage IIIA [30]. Stage IIIA therefore includes at the same time patients with positive peritoneal cytology alone and patients with macroscopic/histological invasion of serosa or adnexal tissues, suggesting that these patients have similar prognosis.

Since then, some studies confirmed the importance of peritoneal cytology by showing that patients with earlystage endometrial cancer with positive peritoneal cytology had worse survival rates than patients with early-stage endometrial cancer with negative peritoneal cytology [3133]. As these studies classified patients according to the clinical staging system [34] (AJCC 2002; based on hysterometry and physical examination), other important prognostic factors like myometrial invasion [35] were not taken into account. As a result, positive cytology appeared 
to be an independent prognostic factor, while in fact, it is more likely a consequence of aggressive or more advanced cancer.

In a recent study by Tebau [36], the 5-year disease-free survival of cytological stage IIIA cancer patients was similar to the one of stage I patients (91 versus 92\%) and better than the one of histological stage IIIA cancer patients. After adjustment for age, myometrial invasion, differentiation, and radiotherapy, cytological stage IIIA patients were still at similar risk to die from endometrial cancer compared to stage I patients.

Similar results are reported by the study of Fadare et al. [37]. According to this study, there is no difference in survival between patients with stage I endometrial cancer and patients with stage IIIA endometrial cancer based on positive peritoneal cytology alone, even after adjusting for other prognostic factors.

This strongly suggests that positive peritoneal cytology is not an independent prognostic factor in patients with endometrial cancer and that positive cytology without other evidence of extrauterine disease leads to upstaging of a minority of endometrial carcinoma patients $(4.5 \%)$, but does not appear to affect their overall outcome.

Generally, most of the recent studies concluded that peritoneal cytology is not an important prognostic factor in endometrial cancer [38-41].

\section{Disseminated malignant cells_-are they viable?}

The reported rate of positive peritoneal cytology in patients with endometrial carcinoma confined to the uterus, and with preceding hysteroscopy and biopsy or curettage, is approximately $12 \%$ [42], compared to a $4.9 \%$ rate without prior hysteroscopy.

In the study of Hirai et al. [43], 50 patients were studied (clinical stage I-II endometrial cancer) with positive peritoneal smears, in which a tube for cytologic analysis was inserted into the peritoneal cavity when closing the abdomen. The peritoneal cavity was irrigated with physiologic saline, and washings were obtained through the tube 7 and 14 days after the operation. On the 14th day of the study, 33 of 34 patients had negative peritoneal cytology. The results of this study suggest that endometrial cancer cells found in the peritoneal cavity usually disappear within a short time and seem to have a low malignant potential.

There is now extensive evidence demonstrating the significance of peritoneal immune mechanisms in the control of metastatic spread [44]. Neoplastic cells' malignant potential is possibly related to the concentrations of malignant cells. The abundance of malignant cells has prognostic importance in identifying those patients with pathologic stage I disease [42]. Questions remain as to whether endometrial cancer cells maintain viability and proliferate once they are displaced into the peritoneal cavity. To answer this question, Arikan et al. [45] performed in vitro hysteroscopy in 24 uteri with endometrial cancer after the specimens were removed surgically. Transtubal fluid dissemination was seen in 20 of 24 cases (83\%). Collected cells were first analyzed with a trypan blue exclusion assay (mean viability, 90\%) then cultured, incubated, and evaluated for adherence to the well, which was a proxy for functional cell viability. Among the disseminated fluid specimens, 17 specimens (71\%) contained tumor cells, of which $42 \%$ demonstrated functional viability. The rate of transtubal spill and malignant cell dispersion can be attributed to the pressure and volume of fluid that was used with hysteroscopy. The results of the study by Arikan et al. demonstrated that disseminated malignant cells were functionally viable in an environment that resembled the peritoneal cavity.

In conclusion, hysteroscopy is highly accurate and clinically useful in the diagnosis of endometrial cancer. There is a slightly higher percentage of positive peritoneal cytology in endometrial cancer patients diagnosed with high-pressure hysteroscopy compared to patients diagnosed with lowpressure hysteroscopy due to transtubal reflux of endometrial cells and tissue into the peritoneal cavity. However, positive peritoneal cytology is not an independent prognostic factor in patients with endometrial cancer and, even if it leads to upstaging of a minority of endometrial carcinoma patients, it does not appear to affect their overall outcome. Moreover, endometrial cancer cells found in the peritoneal cavity in cases of hysteroscopy performed in endometrial cancer patients, although they experimentally show a functional viability, in clinical practice they seem to disappear in a short time and to have a low malignant potential. In cases that endometrial cancer is suspected, spilling can be reduced by a low intrauterine pressure of up to $70 \mathrm{mmHg}$, as tubal dye perfusion in chromopertubation requires a mean pressure of around $100 \mathrm{mmHg}$ [46].

Declaration of interest The authors report no conflicts of interest. The authors alone are responsible for the content and writing of the paper.

\section{References}

1. Loverro G, Bettocchi S, Cormio G et al (1999) Transvaginal sonography and hysteroscopy in postmenopausal uterine bleeding. Maturitas 33:139-144

2. Reis PAS, Nogueira A, Reis FJC et al (2001) Is hysteroscopic appearance a safe mean of diVerentiating malignant from benign 
endometrial polyps in postmenopausal women? Gynaecol Endosc 10:49-51

3. Sugimoto O (1975) Hysteroscopic diagnosis of endometrial carcinoma. A report of fifty-three cases examined at the women's clinic of KyotoUniversity Hospital. Am J Obstet Gynecol 121:105-113

4. Cacciatore B, Ramsay T, Lehtovirta P et al (1994) Transvaginal sonography and hysteroscopy in postmenopausal bleeding. Acta Obstet Gynecol Scand 73:413-416

5. De Wit AC, Vleugels MP, De Kruif JH (2003) Diagnostic hysteroscopy: a valuable diagnostic tool in the diagnosis of structural intra-cavital pathology and endometrial hyperplasia or carcinoma? Six years of experience with non-clinical diagnostic hysteroscopy. Eur J Obstet Gynecol Reprod Biol 110:79-82

6. Iha T, Shen H, Kanazawa K (2003) Hysteroscopy to detect Stage IA well-differentiated endometrioid adenocarcinoma of the endometrium. Acta Obstet Gynecol Scand 82:378-384

7. Garuti G, Mirra M, Luerti M (2006) Hysteroscopic view in atypical endometrial hyperplasias: a correlation with pathologic findings on hysterectomy specimens. J Minim Invasive Gynecol $13: 325-330$

8. Zola FE, Nogueira AA, de Andrade JM et al (2007) Hysteroscopic appearance of malignant and benign endometrial lesions: a casecontrol study. Arch Gynecol Obstet 275(1):49-52

9. Garuti G, De Giorgi O, Sambruni I et al (2001) Prognostic significance of hysteroscopic imaging in endometrioid endometrial adenocarcinoma. Gynecol Oncol 81:408-413

10. Marsahal JL, Rizvi NA, Blargava P et al (2000) J New developmental therapies. Churchil Livingstone, Boston

11. Taddei GIL, Moncini D, Scarselli G et al (1994) Can hysteroscopic evaluation of endometrial carcinoma influence therapeutic treatment? Ann NY Acad Sci 734:482-487

12. Shen H, lha T, Higashi M, Kanazawa K (1999) Preoperative evaluation of myometrial invasion in endometrial carcinoma by magnetic resonance imaging (MRI) and hysteroscopy: diagnostic efficacy of MRI combined hysterectomy. Gynecol Endosc 8:205-212

13. Joja I, Asakawa M, Asakawa T et al (1996) Endometrial carcinoma: dynamic MRI with turbo-FLASH technique. J Comput Assist Tomogr 20:878-887

14. Yamashita Y, Mizutani H, Torashima M et al (1993) Assessment of myometrial invasion by endometrial carcinoma: transvaginal sonography vs contrast enhanced MR imaging. Am J Roentgenol 161:595-599

15. Sironi S, Taccagni G, Garancini P et al (1992) Myometrial invasion by endometrial carcinima: assessment by MR imaging. Am J Roentgenol 158:565-597

16. Gimpelson RJ, Rappold HO (1988) A comparative study between panoramic hysteroscopy with directed biopsies and dilatation and curettage, a review of 276 cases. Am J Obstet Gynecol 152:489-492

17. Garuti G, Sambruni I, Colonnelli M et al (2001) Accuracy of hysteroscopy in predicting histopathology of endometrium in 1500 women. J Am Assoc Gynecol Laparosc 8(2):207-213

18. Clark TJ, Voit D, Gupta JK et al (2002) Accuracy of hysteroscopy in the diagnosis of endometrial cancer and hyperplasia. JAMA 288:1610-1621

19. Lasmar RB, Barrozo PR, de Oliveira MA et al (1996) Validation of hysteroscopic view in cases of endometrial hyperplasia and cancer in patients with abnormal uterine bleeding. Ultrasound Obstet Gynecol 7(6):443-446

20. Gabrielli S, Marabini A, Bevini M et al (1998) Transvaginal sonography vs. hysteroscopy in the preoperative staging of endometrial carcinoma. Eur J Gynaecol Oncol 19(6):561-564

21. Kietlińska Z, Stelmachów J, Antczak-Judycka A et al (2002) Fractional curettage, hysteroscopy and imaging techniques: transvaginal sonography (TVS), magnetic resonance imaging
(MRI) and computed tomography (CT) in the diagnosis of cervical canal involvement in cases of endometrial carcinoma. Int J Gynecol Cancer 12(1):3-17

22. Bartosic D, Jacobs SL, Kelly LJ (1986) Endometrial tissue in peritoneal fluid. Fertil Steril 46:796-800

23. Siegler AM, Lindemann HJ (1984) Hysteroscopy-principles and practice. Lippincot, Philadelphia, pp 145-147

24. Lo KWK, Cheung TH, Yim SF et al (2002) Hysteroscopic dissemination of endometrial carcinoma using carbon dioxide and normal saline: a retrospective study. Gynecol Oncol 84:394-398

25. Nagele F, Wieser F, Deery A et al (1999) Endometrial cell dissemination at diagnostic hysteroscopy: a prospective randomized cross-over comparison of normal saline and carbone dioxide uterine distension. Hum Reprod 14:2739-2742

26. Baker VL, Adamson GD (1995) Threshold intrauterine perfusion pressures for intraperitoneal spill during hydrotubation and correlation with tubal adhesive disease. Fertil Steril 64:10661069

27. Sagawa T, Yamada H, Sakuragi N et al (1994) A comparison between the preoperative and operative findings of peritoneal cytology in patients with endometrial cancer. Asia Oceania J Obstet Gynaecol 20:39-47

28. Solima E, Brusati V, Ditto A et al (2008) Hysteroscopy in endometrial cancer: new methods to evaluate transtubal leakage of saline distension medium. Am J Obstet Gynecol 198:214.e1-214.e4

29. Takac I, Zegura B (2007) Office hysteroscopy and the risk of microscopic extrauterine spread in endometrial cancer. Gynecol Oncol 107(1):94-98

30. Konski A, Poulter C, Keys H et al (1988) Absence of prognostic significance, peritoneal dissemination and treatment advantage in endometrial cancer patients with positive peritoneal cytology. Int J Radiat Oncol Biol Phys 14:49-55

31. Harouny VR, Sutton GP, Clark SA et al (1988) The importance of peritoneal cytology in endometrial carcinoma. Obstet Gynecol 72:394-398

32. Morrow CP, Bundy BN, Kurman RJ et al (1991) Relationship between surgical-pathological risk factors and outcome in clinical stage I and II carcinoma of the endometrium: a Gynecologic Oncology Group Study. Gynecol Oncol 40:55-65

33. Obermair A, Geramou M, Tripcony L et al (2001) Peritoneal cytology: impact on disease-free survival in clinical stage I endometroid adenocarcinoma of the uterus. Cancer Lett 164:105-110

34. AJCC Cancer Staging Handbook (2002) From the AJCC Cancer Staging Manual. Springer, New York

35. Creasman WT, Morrow CP, Bundy BN et al (1987) Surgical pathologic spread patterns of endometrial cancer. A Gynecologic Oncology Group Study. Cancer 60:2035-2041

36. Tebeu PM, Popowski Y, Verkooijen HM et al (2004) Positive peritoneal cytology in early-stage endometrial cancer does not influence prognosis. Br J Cancer 91:720-724

37. Fadare O, Mariappan R, Hileeto D et al (2005) Upstaging based solely on positive peritonealwashing does not affect outcome in endometrial cancer. Mod Pathol 18:673-680

38. Grimshaw RN, Tupper WC, Fraser RC et al (1990) Prognostic value of peritoneal cytology in endometrial carcinoma. Gynecol Oncol 36:97-100

39. Kadar N, Homesley HD, Malfetano JH (1992) Positive peritoneal cytology is an adverse factor in endometrial carcinoma only if there is other evidence of extrauterine disease. Gynecol Oncol 47:145-149

40. Preyer O, Obermair A, Formann E et al (2002) The impact of positive peritoneal washings and serosal and adnexal involvement on survival in patients with stage IIIA uterine cancer. Gynecol Oncol 86:269-273 
41. Kasamatsu T, Onda T, Katsumata N et al (2003) Prognostic significance of positive peritoneal cytology in endometrial carcinoma confined to the uterus. Br J Cancer 88:245-250

42. Szpak CA, Creasman WT, Vollmer RT et al (1981) Prognostic value of cytologic examination of peritoneal washings in patients with endometrial carcinoma. Acta Cytol 25:640-646

43. Hirai Y, Takeshima N, Kato T et al (2001) Malignant potential of positive peritoneal cytology in endometrial cancer. Obstet Gynecol 97:725-728
44. Melichar B, Freedman RS (2006) Immunology of the peritoneal cavity: relevance for host-tumor relation. J Minim Invasive Gynecol 13(5):409-412

45. Arikan G, Reich O, Weiss U et al (2001) Are endometrial carcinoma cells disseminated at hysteroscopy functionally viable? Gynecol Oncol 83:221-226

46. Neis KJ, Brandner P, Keppeler U (1994) Tumor cell seeding caused by hysteroscopy? Geburtshilfe Frauenheilkd 54(12):651655 